FORMATION Formation emploi

Revue française de sciences sociales

112 | octobre-décembre 2010

Pêle-mêle

\title{
Services à la personne : renforcer l'encadrement intermédiaire pour accroítre l'efficacité et l'attractivité des métiers
}

Home care services: strengthening middle management to make the profession more efficient and attractive.

Haushaltsnahe, personenbezogene Dienstleistungen: Förderung der mittleren Führungsebene zur Verbesserung von Effizienz und Attraktivität der betroffenen Berufe

Servicios personales : reforzar el encuadre intermediario para aumentar la eficacia y el atractivo de los oficios

Thérèse Bouvier, Nadine Pelvillain et Paul Santelmann

\section{OpenEdition}

Journals

Édition électronique

URL : http://journals.openedition.org/formationemploi/3187

DOI : 10.4000/formationemploi.3187

ISSN : 2107-0946

Éditeur

La Documentation française

Édition imprimée

Date de publication : 30 décembre 2010

Pagination : 53-67

ISSN : 0759-6340

\section{Référence électronique}

Thérèse Bouvier, Nadine Pelvillain et Paul Santelmann, « Services à la personne : renforcer

l'encadrement intermédiaire pour accroítre l'efficacité et l'attractivité des métiers ", Formation emploi [En ligne], 112 | octobre-décembre 2010, mis en ligne le 10 janvier 2013, consulté le 30 octobre 2020 URL : http://journals.openedition.org/formationemploi/3187 ; DOI : https://doi.org/10.4000/ formationemploi.3187 


\section{Secteur d'activité}

\section{Services à la personne : renforcer l'encadrement intermédiaire pour accroître l'efficacité et l'attractivité des métiers}

Thérèse Bouvier, Nadine Pelvillain, Paul Santelmann*

La fonction d'encadrement intermédiaire répond à trois enjeux : améliorer le service aux clients pour favoriser le développement du secteur, améliorer les conditions de travail par la professionnalisation des aides à domicile, favoriser les évolutions de carrière.

Le développement de l'économie des services, les mutations post-tayloriennes de l'industrie, l'introduction des TIC (technologies de l'information et de la communication) dans la plupart des activités ont modifié les approches métiers classiques. Dans le champ de la formation professionnelle tant initiale que continue, ces évolutions militent pour un dépassement (pas un abandon) de l'analyse de l'emploi sous l'angle du poste de travail ; et ce au profit d'une approche plus globale et plus dynamique qui intègre l'ensemble des emplois concourant à un service ou un produit donné.

Cette approche permet de dépasser une spécialisation et une segmentation de la formation professionnelle par type d'emploi et niveaux de qualification. La formation professionnelle initiale ne peut échapper complètement à une conception gradualiste (par palier ou niveau) de l'apprentissage professionnel ; cependant, les ingénieries de formation d'adultes ont besoin d'une vision plus systémique du travail. Elles ont en
* Thérèse Bouvier est ingénieure de formation sectorielle, spécialisée sur les services à la personne, Direction de I'Ingénierie de I'AFPA (Association nationale pour la formation professionnelle des adultes), therese.bouvier@afpa.fr

Nadine Pelvillain est responsable d'Unité Département Sectoriel Services, Direction de l'Ingénierie de l'AFPA, nadine.pelvillain@afpa.fr

Paul Santelmann est responsable de la Prospective, Direction Générale de I'AFPA. paul.santelmann@afpa. fr. II est chargé d'enseignement à l'université de Paris I (La Sorbonne) et au CNAM (Conservatoire national des arts et métiers). II est notamment l'auteur de: La formation professionnelle continue, collection "Les études» La Documentation française, 2006. «Les distorsions diplômes/qualifications: l'exemple des techniciens et agents de maîtrise de type industriel » in Formation Emploi n 105 janvier - 
mars 2009. "La formation professionnelle tout au long de la vie » in Le système éducatif en France (Dir. B. Toulemonde), "Les Notices ». La Documentation française, 2009. "Place et rôle de l'appareil public de formation » in Encyclopédie de la formation, 2009, PUF, collection "Sciences Humaines ".

effet à répondre à un éventail diversifié d'objectifs (adaptation, perfectionnement, reconversion/mobilité, promotion, élargissement des compétences, polyvalence améliorée, poly et pluri-compétences, etc.) qui suppose une conception plus décloisonnée des qualifications et même des secteurs d'activité. Ainsi, pour établir un référentiel métier de maçon mieux vaut maîtriser également les référentiels d'activité des ouvriers du second œuvre, des chefs de chantiers ou de travaux... L'inverse étant également valable : comment établir les référentiels de compétences des cadres de proximité sans maîtriser les contenus d'activité des ouvriers et des employés?

La conception des référentiels de compétences en formation professionnelle nécessite donc une analyse des organisations de travail et une compréhension fine des fonctions et des compétences mobilisées dans chacun des niveaux opérationnels. C'est d'ailleurs cette analyse qui permet d'acquérir une vision évolutive des qualifications et une grille de lecture de l'impact des innovations techniques et organisationnelles. Une nouvelle technologie ne modifie pas seulement le contenu d'un emploi ou le contour d'une qualification; elle peut transformer une organisation du travail et la répartition des fonctions et des compétences au sein d'un collectif de travail. La diffusion de l'usage des TIC chez les opérateurs a modifié les zones de contrôle et d'influence hiérarchiques. Plus largement, "La circulation des savoirs n'obéit plus à un schéma vertical entre le maître et le compagnon, l'agent de maîtrise et l'ouvrier, le chef de bureau et l'employé. Les organisations tentent désormais de susciter et de canaliser le développement de savoirs expérientiels, individuels et micro-collectifs, comme base d'innovation et d'adaptation à des contraintes d'activité devenues plus mouvantes » (Osty et Uhalde, 2007).

L'étude sur l'encadrement de proximité des services à la personne (SAP), présentée dans cet article, illustre cette exigence méthodologique et le renouvellement et l'enrichissement des études «métiers » de l'AFPA (Association nationale pour la formation professionnelle des adultes) qu'elle a entraînés. Elle s'inscrit dans la vocation de l'AFPA qui vise à qualifier la population active appelée à exercer dans les secteurs dévalorisés et à contribuer à la montée en compétences des individus et des organisations. Le développement quantitatif des SAP est une illustration de l'enjeu de reconnaissance des compétences de services mais aussi du management induit. C'est d'ailleurs toute l'économie " servicielle » qui est marquée par une interrogation forte sur les modes de management (difficulté à définir un équilibre entre prescription des tâches et marge de manœuvre du salarié confronté à la singularité du client). Ce développement des SAP initie également des transformations qualitatives de nombreuses dimensions du rapport qualifications/ compétences/emploi (poids du secteur, nouvelles organisations, exigences montantes des clients, visibilité et lisibilité des compétences mobilisées dans les SAP, scénarios de professionnalisation de l'activité, attractivité du secteur et des fonctions, canaux de recrutement, etc.).

Nos travaux s'inscrivent dans le mouvement de normalisation des métiers de service. Ils visent à mettre en lumière le partage d'un encadrement de l'aide à domicile, porté à la fois par les responsables de secteur et les assistants techniques. De ce point de vue, la professionnalisation des encadrants de proximité est un des leviers (CAS, Métiers 2015, 2007) permettant de sortir les services à domicile de la «non-qualification» attribuée habituellement à ces emplois.

L'objectif est double : définir des certifications professionnelles publiques répondant aux besoins des professionnels, et structurer une offre de formation adaptée. Le secteur des services à domicile dispose, a priori, d'une offre large en termes de certifications professionnelles sur les emplois d'aides à domicile (niveau CAP) et sur les emplois de responsables de secteur (cadres) ; toutefois, l'hypothèse d'une lacune fonctionnelle au niveau de l'encadrement intermédiaire (de proximité) a, jusqu'à présent, été écartée. Notre contribution vise à étayer cette hypothèse en abordant trois questions : comment se situe un emploi d'encadrant de proximité SAP dans le contexte socioéconomique du secteur? Quels sont les contours de cet emploi en termes d'activités et de compétences? 
Comment s'exerce l'encadrement des aides à domicile sous la responsabilité du responsable de secteur? Dans une première partie, nous préciserons les problématiques d'emploi du secteur des SAP. La seconde partie sera centrée sur les représentations négatives attachées à ce secteur. Dans les troisième et quatrième parties, nous explorerons l'enjeu de la professionnalisation et celui de la reconnaissance de la fonction de l'encadrant de proximité dans les services à la personne.

\section{Encadré 1 \\ Le cadre des études de l'AFPA (Association nationale pour la formation professionnelle des adultes)}

Notre étude s'inscrit dans les travaux menés par l'AFPA en matière de veille sectorielle et métiers. Cette veille contribue à alimenter les commissions professionnelles consultatives, chargées d'élaborer les titres professionnels délivrés par le ministère chargé de l'Emploi. Ces études visent notamment à assurer le renouvellement des référentiels des titres existants, en phase avec l'évolution des contenus d'activité des métiers auxquels ils correspondent. Elles ont également une fonction d'anticipation des métiers et fonctions en essor ou en émergence afin d'élaborer de nouveaux titres professionnels.

Les études métiers se fondent sur une combinatoire d'investigations (enquêtes auprès des employeurs, analyse de l'activité selon plusieurs méthodes dont ETED - emploi type étudié dans sa dynamique - qui est un " concept structurant le recueil d'information, l'analyse de cette information, la présentation des résultats et le dialogue entre les personnes chargées de conduire cette analyse et celles qui en attendent un éclairage sur un problème posé dans le champ des ressources humaines 》 (Céreq, Relief $n^{\circ} 14$, 2006). Nos investigations reposent aussi sur une observation et compréhension du passé et du présent (Boyer et Scouarnec, 2009) et prennent en compte la dimension temporelle pour contribuer aux scénarios du futur. Une de nos hypothèses tient dans le constat, pas toujours partagé, que la reconnaissance d'un emploi par une certification professionnelle publique permet de mieux valoriser cet emploi, de faciliter le fonctionnement du marché de l'emploi mais également de contribuer à l'identité économique du secteur d'activité concerné.

S'agissant de l'encadrement de proximité dans les services à la personne, nous avons procédé à 22 entretiens (titulaire du poste $+N+1$ ) dans onze structures représentatives du secteur. L'entretien auprès du supérieur hiérarchique direct a pour objectif d'appréhender l'environnement professionnel du titulaire de l'emploi qui sera ensuite interviewé. Il porte essentiellement sur le champ d'activités, les missions du service, le contexte local et les évolutions récentes, en cours ou annoncées. II permet à la fois de situer l'interviewé dans son collectif de travail, de relever des caractéristiques qui pourront être retenues en tant que facteurs de variabilité et de recueillir des informations qui serviront pour l'analyse des tendances d'évolution. L'entretien dure entre 30 minutes et une heure. II traite :

- De la «sphère professionnelle » sur laquelle le « $n+1$ » exerce son autorité et sa responsabilité : entreprise, division, service, unité, atelier...

- De l'organisation du travail ;

- Des évolutions de l'entreprise (clients, services, gestion, effectifs, etc.) ;

L'entretien auprès du titulaire de l'emploi vise à décrire précisément l'activité et son contexte. II dure environ 1h30. II se décline autour de plusieurs axes:

- La description du travail et de son contexte ;

- Les exigences et les contraintes de l'activité ;

- Les évolutions de l'activité, passées et éventuellement pressenties ;

- Un temps de remarques libres sur l'activité. 


\section{UN SECTEUR EN EXPANSION ET EN RESTRUCTURATION}

Aujourd'hui, le secteur des SAP est hybride entre l'économie marchande et non marchande. Il s'inscrit dans trois sphères : l'économie sociale pour les associations, la sphère publique pour les centres communaux d'action sociale (CCAS) notamment, et la sphère privée pour tous les autres organismes agréés de service à la personne non rattachés aux deux premiers (Adjerad et Gautier, DRTEFP/INSEE, 2009). Son mode de financement reste complexe en raison de l'existence de différents financeurs intervenant selon des logiques diverses : logique d'assurance, de solidarité, logique de prestation de service.
Ce secteur est caractérisé par sa complexité, son hétérogénéité et l'éclatement des acteurs. (Dumalin et Rahou, ANACT, 2008). L'offre de services à la personne est proposée par l'emploi direct (dit «gré à gré ») ou par un organisme, selon trois modes : prestataire (l'organisme est l'employeur de l'intervenant), mandataire (le particulier reste l'employeur de l'intervenant, « placé » par l'intermédiaire d'un organisme), le prêt de main-d'œuvre (mode utilisé par les associations intermédiaires et les entreprises de travail temporaire spécialisées).

Le décret du 26/12/2005 a établi la liste des activités relevant des services à la personne et qui ouvrent la possibilité pour les prestataires d'obtenir un agrément. L'éventail de ces activités (une vingtaine) illustre la difficulté à appréhender l'emploi dans ce secteur

\section{Encadré 2 \\ Le secteur des services à la personne : données chiffrées}

Aujourd'hui, les services à la personne (SAP) se classent au premier rang en termes de création d'emplois. L'observatoire de l'Agence nationale des services à la personne (ANSP) recense, en octobre 2009, 19000 organismes agréés de services à la personne. Entre 2006 et 2009, la répartition est passée de $43 \%$ à $34 \%$ pour les associations, de 35 à $59 \%$ pour les entreprises, de 11 à $7 \%$ pour les établissements publics (centres communaux d'action sociale, CCAS) ; les structures d'insertion professionnelles et autres ont disparu de ce repérage. Quels sont les chiffres clefs de l'emploi, en 2009 ? Selon les prévisions du BIPE (Bureau d'information et de prévision économiques), on dénombre deux millions de salariés dans le secteur (y compris les 309000 assistantes maternelles); plus de $70 \%$ sont employés chez les particuliers employeurs (représentant $45 \%$ des ETP - équivalent temps plein) ; l'emploi prestataire représente $14 \%$ des salariés (près de $19 \%$ des ETP). On compte 1359 millions d'heures travaillées.

La dynamique est forte. Entre 2007 et 2015, la dépense totale pour ce secteur professionnel (hors assistantes maternelles) augmenterait ainsi de 5, 1 milliards d'€ à prix 2007, soit une hausse de $53 \%$ par rapport à 2007. Le marché atteindrait 15 milliards d'euros à prix 2007. En intégrant l'effet de hausses de prix annuelles de l'ordre de $2 \%$ par an en moyenne, cela conduirait à une hausse de $79 \%$ du marché des SAP (hors assistantes maternelles) en huit ans, le marché atteignant 17,2 milliards d' $€$, comparé aux 9,6 milliards d'€ en 2007 (BIPE, 2009).

Le développement de ces services sera important et pourrait représenter $25 \%$ des créations d'emplois d'ici à 2015 (CAS, 2007). Selon l'observatoire de l'ANSP, la croissance de ses effectifs connaît un taux annuel moyen de l'ordre de 5,5\% par an depuis 1990. Elle se poursuit, y compris depuis 2008, avec $+1,5 \%$ pour ce secteur, alors que l'économie globale table sur une régression de plus de $2 \%$ du nombre d'heures travaillées. En effet, le dynamisme de développement de l'emploi est porté par les lourdes tendances démographiques (vieillissement de la population) et par l'évolution des valeurs et des comportements Igénéralisation de l'emploi féminin générant de facto la diminution des aidants familiaux potentie/s). Ces deux mécanismes contribuent à l'essor continu des services hors ou au sein du domicile (Observatoire de la Caisse d'Épargne, 2006). 
qui a généré, en 2008, 796 millions d'heures rémunérées $(+4 \%$ par rapport à 2007$)$ pour 1,3 millions de salariés $(1,6$ million si on intègre la garde des enfants à domicile). $71 \%$ des heures sont réalisées dans le cadre du « gré à gré » (en déclin) contre $29 \%$ qui sont effectuées par des salariés relevant d'organismes prestataires, ce taux étant en hausse (DARES Analyses, 2010).

Ce secteur professionnel est traditionnellement dominé par les associations et les établissements publics. Cependant, la part des entreprises privées (qui ont récemment investi ce marché) monte en puissance au sein des prestataires (Roux, 2007 ; DARES, 2010), même si cette activité reste toujours dominée par le gré à gré, les associations et... le travail non déclaré. Mais tous les segments ne progressent pas à la même vitesse (Bernard, 2007). Les parts de marché des emplois en structures prestataires et mandataires sont appelées à croître au détriment des emplois directs ("gré à gré »). De même, si secteurs privé et associatif poursuivent leur croissance, celle du secteur privé s'avère plus importante (Chol, 2007).

Le développement de la qualité de service est l'enjeu majeur de la maturation et de la croissance de ce secteur professionnel. La pérennisation des emplois est liée à la capacité des structures à garantir la qualité des prestations des services « aide à domicile » (CCIP, 2007). La question de la qualité se pose à deux niveaux dans l'intervention à domicile : garantir l'intervention dans le cadre prévu et la qualité du service rendu. Cette garantie est habituellement portée par la fonction d'encadrement. En effet, la fonction d'encadrant agit sur la mise en place, le suivi et la gestion du service. Or les emplois d'encadrant sont uniquement portés par les structures prestataires et mandataires, et celles-ci accroissent régulièrement leur part de marché. Si les services d'aide à domicile sont bien des services, alors cela réclame, en effet, une structuration des emplois d'encadrant qui prenne en compte la question du coût global du service ne se réduisant pas à la prestation de l'assistant(e). Tous ces points convergent. Ils permettent d'établir que ces emplois d'encadrant intermédiaire vont se développer à la fois de façon proportionnelle mais également suivant une courbe accentuée afin de pallier le déficit actuel de coordination et d'interface dans certaines structures. Développer et professionnaliser les emplois de coordination des intervenants à domicile permet de favoriser les perspectives de mobilité promotionnelle tout en renforçant l'attractivité du secteur qui, de ce fait, devient porteur de perspectives d'évolution professionnelle. L'enjeu est considérable, d'autant qu'un renouvellement générationnel s'annonce dans ce secteur et que l'accroissement du volume de ces emplois est prévisible (CAS Métiers 2015, 2007).

\section{DES REPRÉSENTATIONS NÉGATIVES LIÉES À LA MÉCONNAISSANCE DU TRAVAIL RÉEL}

Le secteur des services d'aide à domicile est confronté, comme nombre de secteurs professionnels centrés sur les activités de services, à une représentation dévalorisée des compétences relationnelles, comportementales ou sociales. Historiquement, les conventions collectives ont d'abord tenu compte de la dimension technique des métiers (liée à l'outillage, aux machines, aux matériaux, aux processus de fabrication, etc.). La hiérarchisation des qualifications inscrite dans les conventions collectives s'est construite sur cette dimension « techniciste"; or, ce modèle n'est pas pertinent pour les métiers de l'aide à domicile où l'outillage ou le produit ne sont pas de mise ou marginaux.

Certes, l'aide à la personne nécessite une dimension technique traditionnelle; cependant, ces métiers ne sont pas réductibles à l'usage de techniques et à la succession de «procédures ». Dans les métiers de l'aide à domicile, l'interaction événementielle (l'assistant à domicile est confronté régulièrement à des événements inattendus, non programmés) et le facteur humain sont prépondérants. Le service se co-construit avec le client (Mayen, 2007).

L'activité d'aide à domicile recouvre également de nombreuses tâches. Celles-ci relèvent toujours d'une double dimension qui peut se résumer à l'adaptation de principes d'action et d'intervention à des contextes particuliers. L'entretien du logement, du linge, la préparation des repas, etc. obéissent à des règles qui paraissent simples au cas par cas. Ces règles deviennent complexes quand il 
s'agit d'intervenir auprès de plusieurs familles ou personnes qui ont des exigences et des habitudes de vie différentes. Un des éléments principaux de la professionnalité des services à domicile tient dans la variété des contextes d'intervention. Dans le cas de l'assistance à des personnes « fragiles 》 ou « diminuées » (jeunes enfants, personnes âgées, malades, handicapés), il s'agit également de maîtriser des invariants en termes de principes d'intervention mais aussi de savoir quand et comment il faut assurer une aide efficace et acceptée par la personne assistée.

Par exemple, un principe de l'aide aux personnes diminuées physiquement consiste à contribuer au maintien ou au développement de leur autonomie physique, intellectuelle ou sociale; cependant, ce principe d'intervention suppose une attention (adaptation au contexte) relative aux moments où il faut les aider. Cette compétence se construit par l'acquisition de règles pratiques et déontologiques et par des mises en situation essentiellement simulées ou reconstituées. Les techniques et les gestes professionnels appropriés dans l'aide à la toilette, à l'habillage, aux déplacements ou à l'alimentation ne vont pas de soi. Elles nécessitent une double compétence : celle liée aux techniques et aux gestes eux-mêmes et celle relative à la capacité à ajuster son intervention lors de la réalisation (DARES, Conditions de Travail, 2005). Dans le champ des services, toute activité exige cette double compétence vérifiable par et dans l'analyse du travail (Hubault et Santelmann, 2007). Or, dans ce secteur, longtemps le travail n'a pas été lisible (Estrade, 2008). S'il commence à l'être au niveau du secteur professionnel, il ne l'est pas encore à l'extérieur. Les représentations négatives sont tenaces. Elles alimentent l'auto-dévaluation des salariés concernés qui n'ont pas l'impression de posséder des savoir-faire réels (ce qui nuit à leur estime de soi et entrave leur évolution professionnelle). "La société a une telle vision de ces emplois de service qu'elle empêche de reconnaître leur qualification réelle de plusieurs manières : la première a trait à la rétribution de cette activité : ni les clients, ni les employeurs ne sont prêts à payer la prestation à son juste prix. Plus subtilement, le statut social de ces emplois interdit aux salariés eux-mêmes d'en percevoir le contenu » (Piotet, 1989). En effet, ce secteur professionnel est né de la transformation des activités de la sphère domestique, mutation économique majeure de la seconde moitié du $\mathrm{xx}^{\mathrm{e}}$ siècle. Une partie des tâches accomplies par les femmes au sein du domicile (les soins apportés aux enfants, personnes âgées, ou malades) est désormais réalisée par des salariés extérieurs de divers services marchands ou non marchands, ce qu'on appelle désormais «services à la personne " (CERC, 2008). Ce secteur professionnel, s'il n'est pas récent, souffre néanmoins des mêmes symptômes que ceux des secteurs en émergence : croissance rapide du secteur conjuguée à des difficultés de recrutement liées à une faible attractivité sectorielle ; déficit d'image des métiers, qualifiés de "petits boulots"; faible rémunération liée à la fréquence du temps partiel; enfin, conditions de travail pénibles croisées avec des politiques publiques privilégiant la croissance quantitative des emplois au détriment des dimensions qualitatives (Dumalin et Rahou ; ANACT, 2008 ; Santelmann, 2002).

Actuellement, la majorité des emplois de ce secteur est tenue par les personnes peu qualifiées ; toutefois, à l'avenir, la concurrence entre les différents segments de l'aide aux personnes peut faire craindre l'accroissement des difficultés de recrutement pour l'aide à domicile, au bénéfice du travail en établissement ou en structure hospitalière. La disparition probable du vivier traditionnel de recrutement des emplois d'aides à domicile, sous l'effet de l'augmentation de la part des femmes titulaires d'un diplôme supérieur ou égal au baccalauréat (entre 1995 et 2015), aura un impact négatif sur l'attractivité de ces métiers. Ils risquent d'être difficiles à pourvoir en dehors d'un recours à l'immigration ou au déclassement, qui est souvent un obstacle à la professionnalisation (volatilité des personnels déqualifiés). Des comportements de fuite vers d'autres secteurs sont également prévisibles (CAS, Métiers 2015, 2007).

L'enjeu est donc de développer l'attractivité de ces métiers par une réflexion sur l'organisation du travail. Il s'agit d'améliorer les conditions de travail, de réduire l'isolement des salariés, de s'attaquer aux faibles rémunérations liées au temps partiel contraint. Une étude régionale récente (INSEE Aquitaine, 2010) fait état de 66800 salariés travaillant dans les services à la personne en 2006 (soit 21 salariés pour 1000 habitants contre 17 en moyenne dans l'ensemble de la France). Or, ramené en équivalent temps plein, 
ce nombre d'emplois équivaut à 22300 ETP. Un des défis de la professionnalisation de ce secteur est donc bien de pallier le manque de perspectives de carrière lié au poids du temps partiel. Professionnalisation du secteur et qualification reconnue des salariés qui y travaillent sont deux enjeux intimement liés.

La professionnalisation ne s'entend donc pas uniquement pour les seuls intervenants à domicile, mais bien pour l'ensemble des salariés contribuant à la réalisation de l'activité. Il s'agit donc tout à la fois de professionnaliser les activités exercées au domicile et celles qui y sont connexes, comme la coordination des intervenants et l'interface avec les demandeurs et les acteurs. La qualité du service d'assistance à domicile passe par le renforcement de l'encadrement intermédiaire (CAS, Métiers 2015, 2007) : la professionnalisation de cette fonction constitue à la fois un enjeu mais aussi, pour une partie du secteur, une obligation. Plus largement, c'est le rapport au client qui est au cœur de cette question : soit le client (la famille ou la personne en demande d'assistance) et l'assistant(e) de vie sont dans une relation bilatérale (" gré à gré »), soit le client a comme interlocuteur une entreprise qui s'engage sur un éventail de prestations référencées et mises en œuvre par un salarié qualifié, c'est-à-dire reconnu comme tel.

De nombreuses entreprises de services à domicile ont désormais intégré la nécessité d'un dialogue avec les clients sur leurs attentes. "La coordinatrice gère la mise en cuvre des missions, réalise les visites au domicile pour évaluer les besoins, gère le dossier de financement, fait également le suivi des interventions, est également présente sur le terrain chaque fois que cela est nécessaire. Elle assure également une charge administrative pure : la réalisation des paies, la facturation aux clients et le recouvrement des impayés » (Mme Z., directrice d'une association intermédiaire d'aide à domicile).

L'encadrement intermédiaire intègre les demandes du client et s'assure de leur appropriation par l'assistant(e) de vie concernée. La régulation et le contrôle ne relèvent plus de la seule relation client/ salarié mais d'une fonction centrale de l'entreprise de service. L'encadrement intermédiaire constitue en cela un maillon structurant de ce secteur. En effet, il organise et coordonne l'activité de l'aide à domicile ; il contribue à l'amélioration de la qualité des services et est à l'interface entre salariés et demandeurs. Dans les grandes agglomérations, là où existe une multiplicité de clients potentiels dans des zones géographiques circonscrites, la nécessité d'une planification et d'une coordination des interventions des assistant(e)s de vie est une évidence. La diversification des prestations, fondée sur un certain niveau de polyvalence des assistant(e)s, apparaît également comme une réponse au temps partiel contraint, souvent basé sur la spécialisation des interventions (la préparation des repas, la garde d'enfants, l'aide à la toilette sont réalisées dans les mêmes tranches horaires...).

D'une part, un meilleur encadrement du métier permet de rassurer le public sur la qualité et le sérieux des prestations proposées à domicile (COCEDAL, 2007) ; d'autre part, la professionnalisation de l'encadrement intermédiaire permettrait d'améliorer les conditions de travail, de limiter l'isolement et de valoriser les compétences des salariés en contact avec les personnes fragiles (CAS, 2007).

\section{UN ENCADREMENT DE PROXIMITÉ, POUR QUOI FAIRE ?}

Arrêtons-nous sur la question de l'encadrement de proximité en général pour nous centrer ensuite sur ce point dans le secteur de l'aide à domicile.

Le coordonnateur d'activité est-il un encadrant? L'organisation du travail réinterroge constamment les niveaux hiérarchiques et fonctionnels. L'encadrement ne concerne pas seulement l'ordre hiérarchique mais s'ouvre largement à d'autres salariés. L'activité professionnelle de ces salariés ne consiste pas à dicter des normes qui structurent l'activité de l'entreprise, mais à les mettre en place, à leur donner forme, voire à les adapter. L'encadrement hiérarchique et l'organisation du travail apparaissent ainsi comme deux formes distinctes d'encadrement. L'un n'implique pas l'autre, même si les deux ont souvent tendance à se cumuler. Par ailleurs, l'encadrement deviendrait une fonction de plus en plus intermédiaire, moins exigeante en diplôme et en qualification (Wolff, 2005). 


\section{Encadré 3}

\section{L'encadrant de proximité, une fonction difficile à cerner...}

Parmi les raisons qui président à la faible émergence des fonctions d'encadrement de proximité des services aux personnes, il y a celle qui tient à la difficulté de nommer ces emplois, comme s'ils étaient dévolus à des personnes dont le secteur ne reconnaît pas vraiment la qualification. En effet, ces emplois ne sont pas nommés précisément dans les conventions collectives. On ne les devine qu'à travers des libellés variés. L'enquête de terrain nous a confronté à des libellés divers: Assistant(e) /Chargé(e) de secteur, Assistant(e) technique, Assistant(e) de direction, Assistant(e) d'agence, Responsable/ Chargé(e) de planning, Coordinateur/trice, Superviseur/seuse, Agent(e) d'accueil et d'accompagnement, Animateur/trice, Secrétaire localle)...

Le répertoire opérationnel des métiers et des emplois (ROME) et la nomenclature des catégories socioprofessionnelles (PCS) de I'INSEE n'identifient pas cette fonction. Les classifications des conventions collectives, diverses et sous-tendues par l'éclatement des statuts des personnes, ne portent pas clairement le repérage de ces emplois. Dans la branche de l'aide à domicile, où sont appliquées de multiples conventions collectives, un accord relatif à l'emploi et aux rémunérations définit notamment les métiers et les répartit en trois filières d'emplois : les personnels d'intervention, les personnels administratifs et de services généraux, les personnels d'encadrement et de direction. L'emploi de responsable de secteur (de niveau scolaire III - BTS/DUT : brevet de technicien supérieur et diplôme universitaire de technologie) est repérable et positionné dans la filière encadrement / direction. Cet emploi n'est reconnu que dans les structures rattachées à cet accord; ; 'exercice de cet emploi entraîne une revalorisation salariale que toutes les structures ne semblent pas en mesure d'honorer.

La Chambre de commerce et d'industrie de la région Centre, recense, en 2006, 17 métiers dans les structures enquêtées, répertoriés à travers deux catégories: les opérationnels et les non-opérationnels qui recouvrent à la fois l'encadrement et le personnel administratif /responsable de secteur/d'équipe, conseiller pédagogique, hôtesse d'accueil, secrétaire polyvalente, assistante, commercial/chargé de développement, contrôleur qualité). Nous constatons que des fonctions et des niveaux très différents sont rassemblés dans une même catégorie.

De leur côté, les établissements publics, pour compléter les emplois fonctionnels de terrain, sont dotés d'une proportion plus importante de personnels administratifs, tandis que les entreprises ont préféré renforcer l'encadrement et le personnel d'interface entre l'exécutant du service et le client. Dans une même catégorie sont regroupés des professionnels n'ayant pas tous le statut de cadre. Sous le terme d'encadrement dans le secteur de l'aide à domicile sont regroupés cadres et non cadres; en témoignent les appellations principales rattachées à la catégorie encadrement : " directeur, directeur adjoint, responsable de service, responsable de secteur, coordinateur de secteur » (Barbe, Coquelle, 2001). Enfin, une enquête auprès des organismes agréés du secteur des services à la personne (Angotti, Olm, Simon, CREDOC, 2007), établit la répartition des personnes physiques, selon les postes occupés, en quatre catégories : le personnel exécutant des prestations de service, le personnel administratif, le personnel assurant l'interface avec les particuliers, les cadres de direction. Seule cette typologie permet de distinguer les encadrants de proximité des cadres de direction.

Accroître la professionnalisation du secteur passe par la prise en compte de l'évolution de l'organisation des structures et des emplois adossés (Croff, 2007). Ici, nous avons choisi de nous centrer sur la question de la professionnalisation des salariés et sur la façon dont elle s'intègre (contribuant de facto à la profes- sionnalisation des organisations). Il convient donc de comprendre comment s'articulent les emplois dans une organisation plus efficace ${ }^{1}$. L'étude a permis de

\footnotetext{
${ }^{1}$ Pour décrire l'emploi, nous nous sommes appuyés sur une analyse des principaux rapports et études sur le secteur, croisée avec une analyse qualitative de l'emploi du secteur (selon une
} 
repérer la distinction et l'articulation des activités du responsable de secteur et de l'encadrant de proximité des aides à domicile.

Les entretiens menés avec des titulaires de l'emploi d'encadrant de proximité éclairent la variété des situations rencontrées, l'importance du travail accompli, mais également la permanence de certaines fonctions... Une encadrante de proximité qui coordonnait, au moment de l'entretien, 37 aides à domicile et 332 usagers, évoquait ses missions : «Je fais la gestion des plannings, du personnel (recrutement, formation, gestion des congés payés, élaboration du contrat de travail : $C D D, C D I$ (contrat à durée déterminée, contrat à durée indéterminée...). Je coordonne les équipes d'aide à domicile sur un même dossier car plusieurs aides à domicile peuvent intervenir chez un même usager. Je définis et redéfinis les politiques de travail (les différentes techniques de travail par rapport aux usagers, la coordination entre elles). C'est beaucoup de bon sens et de cohérence dans leur travail. J'ai les entretiens annuels auprès des 37 aides à domicile. J'assure la gestion du travail en continu, car les aides à domicile travaillent le week-end. J'ai réfléchi à ce problème et il me paraît difficile pour les intervenantes de travailler tout le week-end; aussi, je gère le planning des week end et les aides à domicile travaillent ou le samedi ou le dimanche mais jamais le week-end complet. C'est plus de gestion mais cela me paraît mieux... » $\left(\mathrm{M}^{\mathrm{me}} \mathrm{C}\right.$., 5 ans d'ancienneté dans l'emploi de responsable de secteur, formation initiale : BTS (brevet de technicien supérieur) tourisme, licence en sciences de l'éducation)

D'autres entretiens témoignent de la complexité de la fonction: "Le rôle de l'animatrice est celui de relais, de contrôle auprès des advf (assistantes de vie aux familles) pour vérifier le travail, la qualité de la prestation de l'advf; j'ai aussi un rôle de relais par rapport à la hiérarchie. Mon rôle est donc d'évaluer l'advf au domicile du bénéficiaire, observer sa façon de travailler, d'être... À la fin de l'évaluation, je lis à l'advf son évaluation qu'elle signe. Mais elle peut aussi ajouter un commentaire, parfois elle n'approuve pas tout ce qui est écrit, elle peut justifier ; par exemple, avec l'ADHA, nous avons des tee-shirts

démarche ETED, « Emploi-Type Etudié dans sa Dynamique »cf. encadré méthodologique déjà cité) complétée de rencontres avec les organisations professionnelles. avec le logo, nous devons le porter et si la fille ne le portait pas elle peut se justifier. Cette feuille de suivi, on la donne ensuite à l'infirmière coordonnatrice qui est $M^{\text {me }}$ G., avant il fallait faire une feuille de suivi par fille par mois, maintenant $M^{\text {me }}$ G. a demandé que les animatrices remplissent 2 à 3 feuilles par advf... " $\left(\mathrm{M}^{\mathrm{me}} \mathrm{X}\right.$., un an et demi d'ancienneté dans l'association ; vient de prendre l'emploi d'animatrice après une succession de contrats à temps partiel)

"Le recrutement: on développe en fonction des demandes qui explosent : trouver des gens motivés, qui ont envie, c'est un travail dur, on ne peut s'improviser aide à domicile, il faut de la ponctualité, de l'autonomie, les formations ne sont pas assez nombreuses, la difficulté : trouver des gens qui se sentent valorisés par ce métier. C'est un métier très complexe, on se prend des coups des deux côtés : par les salariés, par les bénéficiaires et leur famille. Etre à l'écoute des demandeurs et des salariés : il faut prendre beaucoup sur soi, rester ferme sur la déontologie, aller au domicile évaluer les besoins, présenter l'association, tenir les plannings à l'heure près : pas de logiciel de planification : j'ai tout dans la tête... Gérer le personnel : les congés, les maladies... Coordonner avec le siège du point de vue juridique. Faire le tampon entre les intervenants et les bénéficiaires pas contents (avoir beaucoup de patience, c'est stressant). Remplacer en urgence, on appelle la famille... » $\left(\mathrm{M}^{\mathrm{me}} \mathrm{D}\right.$., 5 ans d'ancienneté comme encadrante de SAP, après 15 ans de secrétariat, 18 ans d'inactivité pour élever ses trois enfants)

Dans ces organisations du travail, l'encadrant de proximité (pour faciliter la lecture de notre propos nous conviendrons de le nommer "assistant de secteur ») contribue aux activités du responsable de secteur, ce qui permet à ce dernier de se centrer sur les activités qu'il ne peut pas déléguer (Dumalin et Rahou, ANACT, 2008). L'emploi d'assistant de secteur existe en fonction de la taille de la structure et des stratégies d'entreprise. L'élargissement du champ d'intervention des services à domicile (assistance domestique, bricolage, jardinage, etc.) oblige à des réflexions sur la polyvalence des intervenants à domicile, sur la planification des activités, sur la prise en compte de la multi-activité de nombreux salariés qui cumulent des temps partiels dans plusieurs entités et/ 
ou plusieurs clients (gré à gré). Enfin, de nouveaux services (soins esthétiques, assistance informatique) (INSEE Aquitaine, 2010) élargissent la palette organisationnelle et fonctionnelle des entreprises en termes de compétences et d'encadrement.

Cet emploi de coordination n'est pas tenu par un emploi «cadre ». Ainsi les activités de l'assistant de secteur s'articulent, dans les nouvelles organisations du travail, avec celles du responsable de secteur (d'agence) ou du dirigeant de la structure (selon la taille), dont le cœur de métier consiste à encadrer les intervenants au sens hiérarchique du terme.

Aujourd'hui, l'activité du responsable-dirigeant a évolué. Elle est davantage orientée vers les missions de relations avec les clients et donneurs d'ordre, le développement des activités, la conquête de nouveaux clients et la gestion des ressources humaines des équipes. De ce fait, l'assistant de secteur prend en charge une partie de l'activité du responsable concernant :

- L'analyse du besoin des clients ;

- La formalisation et l'évaluation de l'intervention ;

- L'organisation de l'activité des intervenants.

Ainsi, il est l'interface, à un niveau fonctionnel et non hiérarchique, entre le client et l'équipe d'intervenants, sous la responsabilité du responsable de secteur. Il coordonne une équipe d'intervenants et accompagne leur professionnalisation. L'emploi d'assistant de secteur correspond bien à de l'encadrement intermédiaire.

La répartition des activités des deux emplois s'organise dans une logique de complémentarité de compétences. Ce sont les deux volets interactifs d'un même processus (Dumalin et Rahou, ANACT, 2008). Si la logique de compétences est présente, la logique de services est au cœur de la problématique. En effet, les emplois d'aide à la personne sont des emplois où non seulement les compétences relationnelles sont prépondérantes mais où la place du client est beaucoup plus prégnante que dans une entreprise de production de produits manufacturés. Dès lors, comment « industrialise »-t-on un service de proximité ? Pour produire un service en masse, on agit sur les «invariants» (M. Debonneuil, 2007). Est-il possible d'identifier les « invariants » dans une relation de service et si oui, comment agir sur ces « invariants »? En effet, on peut adopter une approche personnalisée de la relation de service. La demande du client est unique, la personne est unique, la perception du besoin également ; la façon de délivrer le service par une approche professionnalisée est plus efficiente.

Quels sont les moyens de construire des invariants ? Organiser les moments de discussion permet de repérer les situations de travail types afin de construire une expérience collective par l'analyse des pratiques et de proposer des solutions types (Ughetto, IRES, 2002). Amener, lors des débriefings des prestations, les aides à domicile à exprimer, à verbaliser leurs prestations peut poursuivre plusieurs objectifs : s'appuyer sur le vécu de la relation, s'extraire de la subjectivité qui pèse toujours dans le relationnel, développer la capacité à repérer comment on interagit pour amener les intervenants à considérer le bénéficiaire des prestations avec la juste distance, réduire la charge mentale des aides à domicile, liée à certaines situations, par une écoute active, relever des indices pour analyser la qualité de la prestation. Bien connaître le client est essentiel ; capitaliser toutes les informations le concernant constitue une plus-value à terme (demandes, besoins, environnement). Recueillir de l'information pour l'entreprise / la structure afin de bien connaître le client et son contexte permet de réaliser les ajustements adéquats par la prise en compte des paramètres les plus pertinents.

Sur cette base, l'entreprise de services aux personnes peut établir des typologies de situations professionnelles ou de contextes par type d'intervention. Un premier volet de cette approche consiste à maîtriser tous les aspects techniques du service à domicile dont le périmètre ne cesse de s'élargir (du repassage au jardinage en passant par les activités de dépannage ou de bricolage qui nécessitent de l'outillage, des compétences techniques, voire des habilitations : par exemple, les interventions touchant l'électricité nécessitent des habilitations professionnelles liées à des formations par des organismes agréés). L'intervention technique suppose des tenues de travail adéquates, des outils ou des produits appropriés, une gestion des stocks, etc. L'assistant de secteur doit savoir maîtriser et contrôler le bon déroulement de cette dimension technique assurée par les intervenant(e)s. Dans un certain nombre de cas, les compétences des services 
à domicile se recoupent avec celles du secteur sanitaire et social (la connaissance basique des propriétés des médicaments utilisés par les personnes âgées fait partie des domaines que l'entreprise d'assistance aux personnes ne peut ignorer) ; cela participe d'ailleurs de cette invisibilité des compétences qui contribue à dévaloriser les assistantes de vie ou les aides-soignantes...

Sous l'angle du rapport direct aux personnes, l'expérience d'un collectif de services aux personnes permet de dépasser la notion de "situations individuelles spécifiques » et de définir des comportements caractéristiques (typologies) en termes :

- D'indépendance des personnes ;

- De sociabilité ;

- D'habitudes culturelles ;

- D'appartenance sociale ;

- D’univers familial ;

- D'habitat et de voisinage ;

- De centres d'intérêt.

Tous ces éléments nécessitent d'être analysés par l'encadrant de proximités et d'être intégrés dans le collectif de travail de l'entreprise et la formation des intervenants. Il s'agit non pas d'aboutir à une standardisation des prestations mais d'éviter les erreurs et mieux préciser préalablement les spécificités du contexte et le cadre d'action de l'intervenant(e). Sur cette base référente, plus ou moins élaborée, l'assistant de secteur, dans son activité d'organisation et de chargé de planning, a pour compétence essentielle de mettre en relation et de réguler les couples «bénéficiaires-intervenants »; compétence comparable à celle des assistants des agences de travail temporaire qui organisent les missions en tenant compte des profils et compétences des intérimaires et des postes à pourvoir. Une connaissance suffisamment fine des questions évoquées ci-dessus, une capacité à détecter les indices révélateurs de difficultés ou de dysfonctionnements en fait un réel manager de proximité du secteur professionnel de l'aide à domicile.

Dans l'analyse du besoin du client potentiel, il y a en effet tout un travail d'identification des éléments particuliers qui vont caractériser l'intervention, éléments qui ne sont pas forcément explicités spontanément par le client. Selon la prestation à mettre en œuvre et à proposer au client, il est donc nécessaire d'avoir une grille de lecture de la situation professionnelle à établir et qui renvoie aux items évoqués plus haut sous l'angle technique et relationnel. La relation au client se consolide également par l'empathie à l'égard de détails de ce type. Elle montre que l'entreprise possède une compréhension de ce qui caractérise une personne, de ce qui est important pour elle : une maison propre et bien rangée témoigne d'un certain rapport à soi différent d'une maison où règne le désordre. La prestation en termes de ménage ou d'entretien doit en tenir compte.

L'expérience de l'entreprise en termes de modification des comportements est également importante. Face à des contextes où l'intervention est difficile (rapport à l'hygiène, à la sécurité, etc., de personnes en voie de dépendance) l'intervenant(e) doit faire usage d'une autorité contrôlée dont il faut évaluer les effets pour «armer» les autres intervenant(e)s. On peut multiplier les exemples où l'analyse des pratiques d'intervention auprès des personnes est nécessaire pour parvenir à un professionnalisme collectif reconnu. L'encadrant de proximité possède un rôle essentiel dans cette évaluation/capitalisation des pratiques qui s'avère un outil de management incontournable.

\section{UN ENJEU DE RECONNAISSANCE ET DE CERTIFICATION}

Cependant, le secteur des services aux particuliers reste le secteur professionnel où le taux de personnel encadrant est le plus faible de tous : $18,6 \%$ en 2010 pour une moyenne de $25,6 \%$. Ce taux évolue très lentement; il était de $16,9 \%$ en 1991. Et si l'on se centre sur la catégorie "employés ", ce taux est encore plus faible. Si 12,7\% des employés en encadrent d'autres, seuls $6,3 \%$ des employés des services aux particuliers ont sous leurs ordres d'autres salariés (DARES, Conditions de Travail, 2005). Ces chiffres témoignent que ce secteur suit la tendance en termes de structuration des emplois mais avec un retard considérable. La principale cause de cette situation est liée au financement même des prestations. Les financements sont en effet basés sur les heures de prestation directe 
au domicile des personnes, majorées forfaitairement pour tenir compte de coûts périphériques actuellement mal mesurés (Dumalin et Rahou, ANACT, 2008). Or, les services à la personne concernent des besoins hétérogènes au sein de multiples domiciles; ce sont des facteurs d'éclatement de l'emploi qui génèrent des problématiques qualitatives nécessitant une régulation spécifique. Il s'agit ici de passer de la juste rémunération de la prestation à la prise en compte du coût global réel d'une structure.

À l'instar des tendances actuelles, où la réduction des lignes et des strates hiérarchiques des emplois tenus par des cadres s'intensifie, on assiste à la lente émergence d'une catégorie d'employés, salariés dans le secteur des aides à domicile, qui organisent le travail des intervenants sans pour autant être identifiés comme encadrants. Cette position est validée par les organisations professionnelles que nous avons rencontrées. Dès les premiers entretiens avec les professionnels (fédérations, syndicats), lorsque nous avons proposé la dénomination de l'emploi sur un libellé d'encadrant de proximité, il est apparu que le terme était en décalage par rapport aux dénominations utilisées dans ce secteur. Pour les responsables nationaux des organisations professionnelles, le responsable de secteur est un encadrant avec un statut cadre, les personnels qui l'aident dans ses missions sont des assistants techniques, voire des assistants d'agence. La profession reste très attachée à une définition « classique », selon laquelle " un encadrant est un cadre »; les techniciens, même détenteurs d'une autorité et exerçant une activité centrée sur l'organisation du travail, n'entrent pas et ne sont pas reconnus dans cette catégorie des encadrants. On est dans un modèle où le cadre statue et où le technicien met en forme la décision (Wolff, 2004).

La montée des exigences des clients, visant notamment la qualité de la relation de service, favorise la nécessaire évolution des organisations. L'emploi d'encadrant de proximité est significatif car il est porteur de la transformation organisationnelle de la structure employeur.

Rappelons que les démarches « qualité » (NF Service « services aux personnes à domicile » de l'AFNOR et la certification "prestations de Services à la personne » de SGS Qualicert - la SGS est aujourd'hui leader mondial en matière de vérification, de contrôles, d'analyses et de certifications) s'imposent au secteur professionnel, quel que soit le statut de l'organisme (privé, public ou associatif). Trois procédures différentes donnent l'agrément simple, l'agrément qualité et l'autorisation (Lesellier, 2007). Elles portent à la fois la nécessité de mettre en place des professionnels assurant suivi et coordination des interventions mais également de les qualifier. L'encadrant est l'un des garants des obligations inhérentes aux normes qualité.

La reconnaissance de ces emplois par une certification lisible est donc posée. En effet, si l'on postule que toute compétence identifiée peut faire l'objet d'une qualification, donc d'une identité professionnelle, alors la reconnaissance la plus aboutie est celle de la certification; celle-ci fournit en effet un signal de reconnaissance sociale permettant une mobilité professionnelle potentielle d'une sphère professionnelle à l'autre et est accessible par l'expérience et par des cursus de formation (Rose, 2009).

En se référant à la nomenclature 1969 des niveaux de formation (même si celle-ci s'avère aujourd'hui une grille de lecture quelquefois inadaptée à la structure des qualifications réelles), l'emploi d'assistant correspond au niveau IV (Bac.). Cette nomenclature précise que "l'activité concerne principalement un travail technique qui peut être exécuté de façon autonome et/ou comporter des responsabilités d'encadrement (maîtrise) et de coordination ».

Les descriptifs du niveau IV du cadre européen des certifications (CEC) (Méhaut et Winch, 2009) apparaissent également pertinents pour définir cet emploi d'assistant de proximité qui doit notamment : "S'autogérer dans la limite des consignes dans des contextes généralement prévisibles mais susceptibles de changer. Superviser le travail habituel d'autres personnes... Etre responsable pour l'évaluation et l'amélioration des activités. »

L'offre de certification foisonne au niveau V (Asseraf, CNCP, 2009) pour les aides à domicile, permettant d'éventuelles mobilités « horizontales »; pour autant, il reste à construire des repères pour des mobilités promotionnelles (verticales), plus difficiles à mettre en œuvre. Le BTS « services et prestations des secteurs sanitaire et social » (niveau III), destiné aux responsables de secteur, est inatteignable par la très grande majorité des salariés de base de ce secteur. 
Quant aux diplômes de niveau IV proches de ce domaine d'activité, ils recouvrent des spécialisations assez éloignées ${ }^{2}$. Il manque à l'évidence une certification de niveau Bac pouvant développer et valider les compétences spécifiques des encadrants/assistants techniques en charge de la coordination des aides à domicile et de l'interface avec les demandeurs.

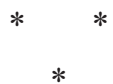

Les résultats de notre étude illustrent, dans le secteur des services à la personne, les contours de l'encadrement intermédiaire (Wolff, 2005) et les compétences qui y sont mobilisées, sous la responsabilité d'un responsable de secteur. Cette fonction permet de répondre à trois enjeux majeurs. Le premier est d'améliorer la qualité du service rendu aux clients pour favoriser le développement du secteur. En effet, le suivi du travail réalisé par les intervenants permet d'accroître l'adéquation des compétences des intervenants aux besoins des clients. Le deuxième enjeu est d'améliorer les conditions de travail par la professionnalisation des aides à domicile (rompre l'isolement, réduire la charge mentale, rationaliser les temps déplacement/temps travail, réduire le temps partiel subi). Enfin, le troisième enjeu est d'ouvrir des perspectives d'évolution de carrière.

En phase avec les besoins et les attentes des professionnels, la création d'une certification professionnelle centrée sur les activités et compétences des emplois d'assistant de secteur/d'agence des services d'aide à la personne de niveau IV (encadrant de proximité) permettrait de reconnaître des compétences professionnelles. Le premier bénéfice serait de reconnaître les personnes occupant l'emploi par

2 Notamment les deux diplômes du ministère des Affaires sociales (les diplômes d'État de Technicien(ne) de l'intervention sociale et familiale et de Moniteur éducateur) et les deux diplômes créés sous l'autorité conjointe du ministère de l'Education nationale et du ministère en charge de l'Agriculture : le Baccalauréat professionnel «Services de proximité et vie locale» (Bac Pro SPVL) et le Baccalauréat professionnel « Services en milieu rural » (Bac pro SMR). la Validation des Acquis de l'Expérience. Le second d'amener de nouveaux actifs sur ce secteur grâce à la perspective d'une mobilité professionnelle horizontale. Le troisième d'attirer des aides à domicile qui, à terme, souhaitent évoluer par une mobilité verticale. Enfin, une certification professionnelle permettrait de construire les parcours de professionnalisation nécessaire pour pouvoir ces emplois.

Cependant, plusieurs questions restent posées. La tentative de reconstruire une lisibilité d'emploi par la reconnaissance des compétences, grâce une certification professionnelle publique, ne soulève-t-elle pas le problème de la déstabilisation des repères qualifications / classifications / certification entreprise depuis vingt ans? Historiquement ancré dans le monde de l'économie sociale, ce secteur basculera-t-il dans l'économie servicielle ? Plus précisément, le développement de l'emploi d'assistant de secteur - en accroissant la capacité des aides à domicile à proposer des services complémentaires et en discernant les besoins de services additionnels, notamment chez les clients « solvables »-sera-t-il un levier de transition (Cohen D., CAS, 2007) ? Plus largement, le cas soulevé par cette fonction d'assistant de secteur qui fait encore débat, fait écho à la difficulté actuelle à reconsidérer l'importance des qualifications intermédiaires dans les mutations du travail, qui voient s'entremêler dans un même emploi des compétences autrefois cloisonnées (Santelmann, 2009). Cette fonction interpelle également l'économie du secteur; de même que la façon dont a été appréhendée la tarification des services à domicile sur la base d'une prestation essentiellement réalisée par une personne et non comme une activité collective intégrant des coûts d'organisation, d'encadrement, de back office, etc. L'encadrement de proximité fait débat. Il illustre en effet la portée économique, concurrentielle et tarifaire d'une activité qui se professionnalise et se structure. Cette professionnalisation a un coût qu'il faudra bien intégrer dans les politiques publiques d'accompagnement de ce secteur composite, dont la pérennité ne fait plus question mais dont la rentabilité demeure insuffisamment étayée. 
Adjerad S. et Gautier C. (2009), Nouveaux regard sur les services à la personne en Haute-Normandie : un secteur dynamique mais un emploi fragile - DTREFP Haute Normandie / INSEE, Aval n ${ }^{\circ} 88$

Aït-Kaci A., Chazalviel A., Colas H., Grapin P., Waelbroeck-Rocha E. (2009), Etude prospective sur les services susceptibles d'émerger dans le secteur des services à la personne, BIPE.

Angotti M., Olm C. et Simon M.-O. (2007), Enquête auprès des organismes agréés du secteur des services à la personne, CREDOC.

Asseraf G. (2009), Pour une simplification de l'offre des certifications dans le champ des services aux personnes fragiles, Rapport au Premier ministre, CNCP.

Barbe L., Coquelle C. (2001), L'encadrement dans le secteur social, juillet, COPAS.

Bernard J. (2007), Les assises des Services à la Personne, Inéum Consulting / ESSEC, mars.

Boyer L. et Scouarnec A. (2009), La prospective des métiers, Éditions EMS.

Caisse d'Épargne (2006), Services à la personne: modes de vie, modes d'emploi, Paris, l'Observatoire Caisse d'Épargne.

Cerc (2008), Les services à la personne, Rapport $\mathrm{n}^{\circ} 8$, Paris, La Documentation française.

Céreq (2006), «La méthode ETED, de l'analyse du travail aux référentiels d'emploi/métiers » Relief Céreq, $\mathrm{n}^{\circ} 14$, février.

Chambre de commerce et d'industrie de Paris (2007), Développement des emplois et des entreprises de service à la personne, Rapport d'étape adopté à l'Assemblée Générale du 7 juin 2007.

Conseil d'Analyse Stratégique (CAS) - DARES (2007), Rapport du groupe PMQ: Les métiers en 2015, Collection «Qualifications \& prospective».
Chol A. (2007), «Les services à la personne en 2005 : poussée des entreprises privées », Premières synthèses, $\mathrm{n}^{\circ} 20.1$, mai, DARES.

Cocedal Conseil (2007), Enquête Nationale auprès des Organismes Agréés de Services à la PersonneAnalyse des acteurs du marché, novembre, ANSP.

Cohen D. (2009), Sortie de crise: Vers l'émergence de nouveaux modèles de croissance?, Rapport CAS document de travail, www.strategie.gouv.fr.

Croff B. (2007), «La GRH demeure une question cruciale, Professionnaliser les services à la personne », Développement, ${ }^{\circ} 45$, février.

DARES (2010), «Les services à la personne: une croissance vive en 2007, atténuée en 2008 », Analyses, $\mathrm{n}^{\circ}$ 020, avril

Debonneuil M. (2007) «L'espoir économique : vers la révolution du quaternaire», Économie $d u$ Quaternaire, Édition Bourrin.

Dumalin F. et Rahou N. (2008), Services à la personne: Évolutions, organisation et conditions de travail, Étude et documents, ANACT, Lyon.

DARES (2007), Enquêtes Conditions de travail 1984-2005 : résultats détaillés, complément de l'enquête Emploi de l'INSEE en continu 2007.

Estrade M.-A. (2008), «Une prospective socioéconomique du travail et de l'emploi peu qualifié », L'emploi, nouveaux enjeux, INSEE références, pp. 27-39.

Hubault F. et Santelmann P. (2007), Les ambivalences du travail non qualifié, une lecture du travail en actes, rapport du groupe Prospective des métiers et des qualifications, CAS.

INSEE Aquitaine (2010), « D'ici 2020, des emplois aquitains à créer dans les services à la personne », 4 pages, septembre. 
Lesellier J.-N. (2007), Les services à la personne: comment ça marche, Wolters Kluwer, France, novembre.

Mayen P. (2007), «Quelques repères pour analyser les situations dans lesquelles le travail consiste à agir pour et avec un autre », cahier $d u C R E N, 4$.

Méhaut Ph. et Winch Ch. (2009), "Le cadre européen des certifications, quelles stratégies nationales d'adaptation? », Formation Emploi n ${ }^{\circ} 108$.

Observatoire prospectif des besoins en compétences, qualifications et formations (2006), Les services à la personne, édition 2006-2007, Chambre régionale de Commerce et d'Industrie Centre.

Osty F. et Uhalde M. (2007), Les mondes sociaux de l'entreprise, Éditions La Découverte.

Piotet F. (1989), "Qualifiés fast-food», Revue PROJET, $\mathrm{n}^{\circ} 218$.

Roux T. (Dir.) (2007), Les stratégies dans les services à la personne, le secteur privé au défi de la professionnalisation, PRECEPTA - Groupe Xerfi.
Rose J. (2009), La "non qualification» question de formation, d'emploi ou de travail ?, Céreq, net. doc 53 .

Santelmann P. (2002), Qualification ou compétences, en finir avec la notion d'emplois non qualifiés, Éditions Liaisons, collection «Entreprise \& Carrières ».

Santelmann P. (2009), «Les distorsions diplômes/ qualifications : l'exemple des techniciens et agents de maîtrise de type industriel », Formation Emploi $\mathrm{n}^{\circ} 105$.

Ughetto P. (2002), Compétence de service : état des lieux d'une problématique, Document de travail $\mathrm{n}^{\circ}$ 02.03, IRES.

Wolff L. (2005), Transformations de l'intermédiation hiérarchique, Rapport de recherche du Centre d'Etude de l'Emploi, nº 29.

Wolff L. (2004), Qu'est-ce qu'encadrer veut dire? Cadres, managers et personnel d'encadrement, La «jeune recherche», Actes d'une journée organisée par le GDR C.A.D.R.E.S.), « Cahier du GDR C.A.D.R.E.S », pp. 104-114.

\section{Résumé}

\section{Services à la personne : renforcer l'encadrement intermédiaire pour accroître l'efficacité et l'attractivité des métiers}

Thérèse Bouvier, Nadine Pelvillain, Paul Santelmann

L'étude éclaire le partage de l'encadrement de l'aide à domicile, entre les responsables de secteur d'une part et les assistants techniques d'autre part. La question de la lisibilité des compétences des uns et des autres est au cœur de cet article. La création d'une certification professionnelle ciblée contribuerait à objectiver et à valoriser les fonctions des encadrants pour résoudre les questions de professionnalisation et d'attractivité de ce secteur professionnel.

Mots clés :

Service aux particuliers, aide à domicile, encadrement, certification, professionnalisation, compétence Journal of Economic Literature: M 51, J 24 